\title{
How to Detect and Remove Temporal Autocorrelation in Vehicular Crash Data
}

\author{
Azad Abdulhafedh* \\ University of Missouri-Columbia, MO, USA \\ Email: asa8cd@mail.missouri.edu
}

How to cite this paper: Abdulhafedh, A. (2017) How to Detect and Remove Temporal Autocorrelation in Vehicular Crash Data. Journal of Transportation Technologies, 7, 133-147.

https://doi.org/10.4236/jtts.2017.72010

Received: February 12, 2017

Accepted: April 14, 2017

Published: April 17, 2017

Copyright $\odot 2017$ by author and Scientific Research Publishing Inc. This work is licensed under the Creative Commons Attribution International License (CC BY 4.0).

http://creativecommons.org/licenses/by/4.0/ (c) (7) Open Access

\begin{abstract}
Temporal autocorrelation (also called serial correlation) refers to the relationship between successive values (i.e. lags) of the same variable. Although it has long been a major concern in time series models, however, in-depth treatments of temporal autocorrelation in modeling vehicle crash data are lacking. This paper presents several test statistics to detect the amount of temporal autocorrelation and its level of significance in crash data. The tests employed are: 1) the Durbin-Watson $(D W) ; 2)$ the Breusch-Godfrey $(L M)$; and 3) the Ljung-Box $Q(L B Q)$. When temporal autocorrelation is statistically significant in crash data, it could adversely bias the parameter estimates. As such, if present, temporal autocorrelation should be removed prior to use the data in crash modeling. Two procedures are presented in this paper to remove the temporal autocorrelation: 1) Differencing; and 2) the Cochrane-Orcutt method.
\end{abstract}

\section{Keywords}

Serial Correlation, Durbin-Watson, Breusch-Godfrey, Ljung-Box, Differencing, Cochrane-Orcutt

\section{Introduction}

Temporal autocorrelation (i.e. serial correlation) is a special case of correlation, and refers not to the relationship between two or more variables, but to the relationship between successive values of the same variable. Temporal autocorrelation is closely related to the correlation coefficient between two or more variables, except that in this case we do not deal with variables $X$ and $Y$, but with lagged values of the same variable. Most regression methods that are used in crash modeling assume that the error terms are independent from one another, 
and they are uncorrelated. This assumption is formally expressed [1] as:

$$
E\left(\varepsilon_{i} \varepsilon_{j}\right)=0.0 \text { for all } i \neq j
$$

where,

$E$ : the expected value of all pair-wise products of error terms,

$\varepsilon_{i} \varepsilon_{j}$ : error terms of the $i$ and $j$ observations respectively,

which means that the expected value of all pair-wise products of error terms is zero, and when the error terms are uncorrelated, the positive products will cancel those that are negative leaving an expected value of 0.0 [1]. If this assumption is violated, the standard errors of the estimates of the regression parameters are significantly underestimated which leads to erroneously inflated coefficients values, and incorrect confidence intervals. The presence of correlated error terms means that these types of inferences cannot be made reliably [2]. The violation of this assumption occurs because of some temporal (time) component (i.e. heterogeneity due to time) that can affect the observations drawn across the time, such as time series data, panel data in the form of serial correlation, and any other dataset that might be collected over a period of time. In this context, the error in a first time period influences the error in a subsequent time period (either the previous period, or the next period or beyond) [3]. For example, we might expect the disturbance (i.e. error term) in year $t$ to be correlated with the disturbance in year $t-1$ and with the disturbance in year $t+$ $1, t+2$, and so on. If there are factors responsible for inflating the observation at some point in time to an extent larger than expected (i.e. a positive error), then it is reasonable to expect that the effects of those same factors linger creating an upward (positive) bias in the error term of a subsequent period. This phenomenon is called positive first-order autocorrelation, which is the most common manner in which the assumption of independence of errors is violated. For instance, if a dataset influenced by quarterly seasonal factors, then a resulting model that ignores the seasonal factors will have correlated error terms with a lag of four periods. There are different structure types of temporal autocorrelation: $1^{\text {st }}$ order, $2^{\text {nd }}$ order, and so on. The form of temporal autocorrelation that is encountered most often is called the first order temporal autocorrelation in the first autoregressive term, which is denoted by AR (1). The AR (1) autocorrelation assumes that the disturbance in time period $t$ (current period) depends upon the disturbance in time period $t-1$ (previous period) plus some additional amount, which is an error, and can be modeled as [3]:

$$
\varepsilon_{t}=\rho \varepsilon_{t-1}+\epsilon_{t}
$$

where,

$\varepsilon_{t}:$ the disturbance in time period $t$,

$\varepsilon_{t-l}:$ the disturbance in time period $t-1$,

$\rho$ : the autocorrelation coefficient,

$\epsilon_{i}$ : the model error term.

The parameter $\rho$ can take any value between negative one and positive one. If $\rho>0$, then the disturbances in period $t$ are positively correlated with the disturbances in period $t-1$. In this case, positive autocorrelation exists which means 
that when disturbances in period $t-1$ are positive disturbances, then disturbances in period $t$ tend to be positive. When disturbances in period $t-1$ are negative disturbances, then disturbances in period $t$ tend to be negative. Temporal datasets are usually characterized by positive autocorrelation. If $\rho<0$, then the disturbances in period $t$ are negatively correlated with the disturbances in period $t-1$. In this case there is negative autocorrelation. This means that when disturbances in period $t-1$ are positive disturbances, then disturbances in period $t$ tend to be negative. When disturbances in period $t-1$ are negative disturbances, then disturbances in period $t$ tend to be positive.

The second order temporal auto correlation is called the second-order autoregressive process or AR (2). The AR (2) autocorrelation assumes that the disturbance in period $t$ is related to both the disturbance in period $t-1$ and the disturbance in period $t-2$, and can be modeled as [3]:

$$
\varepsilon_{t}=\rho_{1} \varepsilon_{t-1}+\rho_{2} \varepsilon_{t-2}+\epsilon_{t}
$$

where,

$\rho_{1}$ : the autocorrelation coefficient in time period $t-1$.

$\rho_{1:}$ the autocorrelation coefficient in time period $t-2$.

The disturbance in period $t$ depends upon the disturbance in period $t-1$, the disturbance in period $t-2$, and some additional amount, which is an error $\left(\epsilon_{t}\right)$. In a similar manner, the temporal autocorrelation can be extended to the $\rho$ th order autocorrelation AR $(\rho)$. However, the most often used temporal autocorrelation is the first-order autoregressive process [3]. If the temporal autocorrelation is found to be significant in crash data, then it must be removed before using the data in the modeling process [4] [5] [6].

\subsection{Sources of Temporal Autocorrelation}

Temporal autocorrelation can arise from the following sources:

- Omitted Explanatory Variables: Omitting some important explanatory variables from the modeling process can create temporal autocorrelation that can produce biased parameter estimates and incorrect inferences, especially if the omitted variable is correlated with variables included in the model [1] [7] [8] [9].

- Misspecification of the Mathematical Form of the model can create temporal autocorrelation. For example, if a linear form of the model is specified when the true form of the model is non-linear, the resulting errors may reflect some temporal autocorrelation [10] [11] [12] [13].

- Misspecification of The Error Terms of the model due to some purely random factors, such as changes in weather conditions, economic factors, and other unaccounted for variables, which could have changing effects over successive periods. In such instances, the value of the error terms in the model could be miss pecified [3].

\subsection{Detection of Temporal Autocorrelation}

Several methods are available to detect the existence of the temporal autocorrela- 
tion in the crash dataset, including the residuals scatter plots, the Durbin-Watson test, the Durbin h test, the Breusch-Godfrey test, the Ljung-Box $Q$ test, and correlograms. These will be described in detail below:

\section{- Scatter Plot of Residuals}

The error for the $i^{\text {th }}$ observation in the dataset is usually unknown and unobservable. However, the residual for this observation can be used as an estimate of the error, then the residuals can be plotted against the variables that may be related to time. The residual would be measured on the vertical axis. The temporal variables such as, years, months, or days would be measured on the horizontal axis. Next, the residual plot can be examined to determine if the residuals appear to exhibit a pattern of temporal autocorrelation. If the data are independent, then the residuals should be randomly scattered about 0.0 . However, if a noticeable pattern emerges (particularly one that is cyclical or seasonal) then temporal autocorrelation is likely an issue. It must be emphasized that this is not a formal test of serial correlation. It would only suggest whether temporal autocorrelation may exist. We should not substitute a residual plot for a formal test [1] [13].

\section{- The Durbin-Watson (DW) Test}

The most often used test for first order temporal autocorrelation is the Durbin-Watson $D W$ test [13]. The $D W$ test is a measure of the first order autocorrelation and it cannot be used to test for higher order temporal autocorrelation. The $D W$ test is constructed to test the null and alternative hypotheses regarding the temporal autocorrelation coefficient $(\rho)$ :

$$
H_{0}: \rho=0.0, H_{a}: \rho \neq 0.0
$$

The null hypothesis of $\rho=0.0$ means that the error term in one period is not correlated with the error term in the previous period, while the alternative hypothesis of $\rho \neq 0.0$ means the error term in one period is either positively or negatively correlated with the error term in the previous period. To test the hypothesis, the $D W$ test statistic on a dataset of size $n$ is formulated as [1]:

$$
D W=\frac{\sum_{t=2}^{n}\left(e_{t}-e_{t-1}\right)^{2}}{\sum_{t=1}^{n} e_{t}^{2}}
$$

where,

$D W$ : the Durbin-Watson statistic,

$e_{t}$ : the residual error term in time period $t$,

$e_{t-1}$ : the residual error term in the previous time period $t-1$.

The $D W$ statistics ranges from 0.0 to 4.0 , and it can be shown that:

$$
D W=2\left(1-\rho^{\wedge}\right)
$$

where,

$\rho^{\wedge}$ : the residual temporal autocorrelation coefficient.

When $\rho^{\wedge}=0.0$, (i.e. no autocorrelation), then $D W=2.0$.

When $\rho^{\wedge}$ tends to 1.0 , then $D W=0.0$.

When $\rho^{\wedge}$ tends to -1.0 , then $D W=4.0$.

The critical values of $D W$ for a given level of significance, sample size and number of independent variables can be obtained from published tables that 
are tabulated as pairs of values: DL (lower limit of $D W$ ) and DU (upper limit of $D W)$. To evaluate $D W[3]$ :

1) Locate values of DL and DU in Durbin-Watson statistic table.

2) For positive temporal autocorrelation:

a) If $D W<\mathrm{DL}$ then there is positive autocorrelation.

b) If $D W>\mathrm{DU}$ then there is no positive autocorrelation.

c) If $\mathrm{DL}<D W<\mathrm{DU}$ then the test is inconclusive.

3) For negative temporal autocorrelation:

a) If $D W<(4.0-\mathrm{DU})$ then there is no negative autocorrelation.

b) If $D W>(4.0-\mathrm{DL})$ then there is negative autocorrelation.

c) If $(4.0-\mathrm{DU})<D W<(4.0-\mathrm{DL})$ then the test is inconclusive.

A rule of thumb that is sometimes used is to conclude that there is no first order temporal autocorrelation if the $D W$ statistic is between 1.5 and 2.5. A $D W$ statistic below 1.5 indicates positive first order autocorrelation. A $D W$ statistic of greater than 2.5 indicates negative first order autocorrelation [3]. Alternatively, a significant $p$-value for the $D W$ statistic would suggest rejecting the null hypothesis and concluding that there is first order autocorrelation in the residuals, and a non-significant $p$-value would suggest accepting the null hypothesis and concluding that there is no evidence of first order autocorrelation in the residuals.

\section{- The Durbin $h$ Test}

When one or more lagged dependent variables are present in the data, the $D W$ statistic will be biased towards 2.0 , this means that even if temporal autocorrelation is present it may be close to 2.0, and hence it cannot detect it. Durbin suggests a test for temporal autocorrelation when there is a lagged dependent variable in the dataset, and it is based on the $h$ statistics. The Durbin $h$ statistics is defined as:

$$
h=\rho^{\wedge} \sqrt{\frac{T}{1-T\left[\operatorname{VAR}\left(\beta^{\wedge}\right)\right]}}
$$

where,

T: the number of observations in the dataset,

$\rho^{\wedge}$ : the temporal autocorrelation coefficient of the residuals,

$\operatorname{VAR}\left(\beta^{\wedge}\right)$ : the variance of the coefficient on the lagged dependent variable.

Durbin has shown that the $h$ statistics is approximately normally distributed with a unit variance, hence the test for first order autocorrelation can be done using the standard normal distribution. If Durbin $h$ statistic is equal to or greater than 1.96 , it is likely that temporal autocorrelation exists [1].

\section{- The Breusch-Godfrey Lagrange Multiplier (LM) Test}

The Breusch-Godfrey test is a general test of serial correlation and can be used to test for first order temporal autocorrelation or higher order autocorrelation. This test is a specific type of Lagrange Multiplier test. The $L M$ test is particularly useful because it is not only suitable for testing for temporal autocorrelation of any order, but also suitable for models with or without lagged dependent variables [14]. The null and alternative hypotheses used with this test for a second order autocorrelation are: 


$$
H_{0}: \rho_{1}=\rho_{2}=0.0, H_{1}: \text { At least one } \rho \text { is not zero }
$$

The $L M$ test statistic is given by:

$$
L M=(n-i) R^{2}
$$

where,

LM: the Lagrange multiplier test statistic,

$n$ : the number of observations in the dataset,

$i$ : the order of the autocorrelation,

$R^{2}$ : the unadjusted $R^{2}$ statistic (coefficient of determination) of the model.

The $L M$ statistic has a chi-square distribution with two degrees of freedom, $\chi^{2}$ (2) $[15]$.

\section{- The Ljung-Box $Q$ (LBQ) Test}

The Ljung-Box $Q$ test (sometimes called the Portmanteau test) is used to test whether or not observations taken over time are random and independent for any order of temporal autocorrelation. It is based on asymptotic Chi-Square distribution $\chi^{2}$. In particular, for a given $i$ lag, it tests the following hypotheses [16]:

$H_{0}$ : the autocorrelations up to $i$ lags are all zero

$H_{a}$ : the autocorrelations of one or more lags differ from zero

The test statistic is determined as follows [16]:

$$
L B Q_{i}=n(n+2) \sum_{j=1}^{i} \frac{r_{j}^{2}}{n-j}
$$

where,

$L B Q_{i}$ : the Ljung-Box $Q$ statistic,

$n$ : the number of observations in the data,

$j$ : the lag being considered,

$i$ : the autocorrelation order,

$r$. the residual error term in lag $j$.

\section{- Correlograms}

Correlograms are autocorrelation plots that can show the presence of temporal autocorrelation. The autocorrelation would appear in lag 1.0 and progress for $\mathrm{n}$ lags then disappear. In these plots the residual autocorrelation coefficient $\left(\rho^{\wedge}\right)$ is plotted against $n$ lags to develop a correlogram. This will give a visual look at a range of autocorrelation coefficients at relevant time lags so that significant values may be seen [17]. In most software packages, two types of autocorrelation functions are presented: the autocorrelation function (ACF), and the partial autocorrelation function (PACF). The ACF is the amount of autocorrelation between a variable and a lag that is not explained by correlations at all lower-orderlags, and the PACF is the difference between the actual correlation at specific lag and the expected correlation due to propagation of correlation at the previous lag. If the PACF displays a sharp cutoff while the ACF decays more slowly we conclude that the data displays an autoregressive model (AR), and the lag at which the PACF cuts off is the indicated number of AR terms. If the ACF of the data displays a sharp cutoff and/or the lag-1 autocorrelation is negative then we have to consider adding a moving average term (MA) to the model, and the lag at which the ACF cuts off is the indicated number of MA terms. In general, the 
diagnostic patterns of ACF and PACF for an AR (1) term [18] are:

ACF: declines in geometric progression from its highest value at lag 1.0.

PACF: cuts off abruptly after lag 1.0.

If the $\mathrm{ACF}$ of a specific variable shows a declining geometric progression from the highest value at lag 1.0, and the PACF shows an abrupt cut off after lag 1.0., this would indicate that this variable has not encountered temporal autocorrelation.

\subsection{Remedies for Temporal Autocorrelation}

When temporal autocorrelation is determined to be present in the dataset, then one of the first remedial measures should be to investigate the omission of one or more of the key explanatory variables, especially variables that are related to time. If such a variable does not aid in reducing or eliminating temporal autocorrelation of the error terms, then a differencing procedure should be applied to all temporal independent variables in the dataset to convert them into their differences values, and rerun the regression model by deleting the intercept from the model [17]. If this remedy does not help in eliminating temporal autocorrelation, then certain transformations on all variables can be performed for the AR (1) term. These transformations aim at performing repeated iterative steps to minimize the squared sum of errors in the regression model. Examples of such transformations are: Cochrane-Orcutt procedure; and Hildreth-Lu procedure. More advanced methods can also be used for big datasets such as: Fourier series analysis; and the spectral analysis [17] [18].

\section{Data}

Missouri crash data for three years (2013-2015) for the Interstate I-70, MO, USA are used in this paper as reported by the Missouri State Highway Patrol (MSHP) and recorded in the Missouri Statewide Traffic Accident Records System (STARS). The data included a wide range of independent variables (i.e. risk factors) in the analysis:

- Road geometry (grade or level; number of lanes)

- Road classification (rural or urban; existing of construction zones)

- Environment (light conditions)

- Traffic operation (annual average daily traffic, AADT)

- Driver factors (driver's age; speeding; aggressive driving; driver intoxicated conditions; the use of cell phone or texting)

- Vehicle type (passenger car; motorcycles; truck)

- Number of vehicles involved in the crash

- Time factors (hour of crash occurrence; weekday; month)

- Accident type (animal; fixed object; overturn; pedestrian; vehicle in transport).

\section{Methodology}

In this paper, three of the most widely used tests to detect the existence of tem- 
poral autocorrelation in the crash data are investigated, namely: The DurbinWatson $(D W)$, the Breusch-Godfrey $(L M)$, and the Ljung-Box $Q(L B Q)$ tests. The three temporal independent variables in the dataset (i.e. month, weekday, hour) are used in the application of each test.

The tests can be applied at different levels of temporal aggregation (i.e. over one year, over two years, three years, etc.) to help identify any hidden effects of the temporal autocorrelation that might exist within a timeframe. In this paper, the JMP12 software package is used to compute the $D W$ statistics, the associated residual temporal autocorrelation coefficients, and their significance at the $95 \%$ confidence level (i.e. $p$-values). JMP requires that the input format of the crash data be in either excel spreadsheet (i.e. ${ }^{*}$.xlsx) or in text (i.e. delimited or ${ }^{*}$.csv) and then the output is produced as excel spreadsheet or delimited text. The Eviews 9 software is used to compute the $L M$ statistics, and their significance at the $95 \%$ confidence level (i.e. $p$-values). The software requires that the input format of the crash data be in either excel spreadsheet (i.e. ${ }^{*}$.xlsx) or in text (i.e. delimited or ${ }^{*}$.csv) and then the output is produced as excel spreadsheet or delimited text. The Stata 14 software is used to compute the Box-Ljung $Q$ statistic $(L B Q)$ at each lag separately with the autocorrelation function $(A C F)$ and the partial autocorrelation function $(P A C F)$ at each lag as well, and their significance at the $95 \%$ confidence level (i.e. $p$-values). The software requires that the input format of the crash data be in either excel spreadsheet (i.e. ${ }^{*}$.xlsx) or in text (i.e. delimited or ${ }^{*}$.csv) and then the output is produced as excel spreadsheet or delimited text.

The Durbin Watson $(D W)$ test is applied to the I-70 data at two temporal levels; aggregation by year, and aggregation over all three years. Data for each year in aggregate is separately tested using (month, weekday, and hour) as the independent temporal variables, and then the aggregate three-year period is tested using the same independent variables.

The Breusch-Godfrey ( $L M$ ) test is applied to the I-70 data for the first 36 lags at two temporal levels; aggregation by year, and aggregation over all three years. Data for each year in aggregate is separately tested using (month, weekday, and hour) as the independent temporal variables, and then the aggregate three-year period is tested. The $L M$ test is applied with degrees of freedom equal to the number of lags (i.e. 36 degrees of freedom). The minimum recommended number of lags that should be considered for the $L M$ and $L B Q$ tests is roughly taken as the natural logarithm of the number of observations within the dataset [19], and larger values are recommended to detect the existence of temporal autocorrelation. For the I-70 dataset, the number of observations of the aggregated three years (2013-2015) is 5869, and the minimum recommended number of lags $=\ln$ $(5869)=8.7$. This paper uses 36 lags in both the $L M$ and $L B Q$ tests instead of the minimum recommended number.

The Box-Ljung $Q$ statistic ( $L B Q$ ) is applied to the I-70 data for the aggregated three-year period (2013-2015) using the time independent variables (month, weekday, and hour) and for the first 36 lags. In addition, correlograms of the 
autocorrelation function (ACF) and partial autocorrelation function (PACF) for the I-70 data for the aggregated three-year period (2013-2015) are presented.

\subsection{The Durbin-Watson Test Results}

Table 1 shows the results of the Durbin-Watson $(D W)$ test for the I-70 at the one-year aggregate level. It can be seen that the temporal autocorrelation of the I-70 dataset for the year 2013 is found to be $3.64 \%$ with $p$ value of 0.0512 (which is non-significant at alpha of 0.05); for the year 2014 year is found to be $7.19 \%$ with $p$-value of 0.0002 (which is significant at alpha of 0.01 ); and for the year 2015 is found to be $2.38 \%$ with $p$-value of 0.1371 (non-significant at alpha of $0.05)$. So, the only significant temporal autocorrelation is existed within the I-70 (2014) data, which should be removed before using this dataset in any modeling process.

\subsection{The Breusch-Godfrey Test Results}

Table 2 shows the results of the $L M$ test for the I-70 crash data at the one-year aggregate level. The $L M$ value (using 36 lags or 36 degrees of freedom) of the I-70 dataset for the year 2013 is found to be 31.022 with $p$-value of 0.7042 (nonsignificant at alpha of 0.05); for the year 2014 is found to be 60.129 with $p$-value of 0.0071 (significant at alpha of 0.01); and for the year 2015 is found to be 50.876 with $p$-value of 0.0512 (non-significant at alpha of 0.05 ). The results of the $L M$ test confirm the results of the $D W$ test that the I-70 dataset for the year 2014 contains a significant temporal autocorrelation as shown in Table 2.

\subsection{Removal of the Temporal Autocorrelation from Crash Data}

Since both the DW and the LM tests have shown the existence of temporal autocorrelation in the I-70 (2014) crash data, the next step is to remove it before using the data in any modeling process. Two approaches are investigated in this paper for the removal of temporal autocorrelation, the differencing procedure, and the Cochrane-Orcutt procedure.

Table 1. DW statistic for I-70 crash data.

\begin{tabular}{ccccc}
\hline Year & Durbin-Watson (DW) & Temporal Autocorrelation Coefficient & P-value & Decision \\
\hline 2013 & 1.927 & 0.0364 & 0.0512 & non-sig \\
2014 & 1.843 & 0.0719 & 0.0002 & sig. \\
2015 & 1.952 & 0.0238 & 0.1371 & non-sig \\
\hline
\end{tabular}

Table 2. LM statistic for I-70 crash data.

\begin{tabular}{cccc}
\hline Year & LM statistic & p-value & Decision \\
\hline 2013 & 31.022 & 0.7042 & non-sig \\
2014 & 60.129 & 0.0071 & Sig. \\
2015 & 50.876 & 0.0672 & non-sig \\
\hline
\end{tabular}




\subsection{The Differencing Procedure}

Since a significant temporal autocorrelation is found to be existed within the I70 (2014) data, then this should be removed before using the dataset in any potential modeling process [4] [5] [6]. In order to remove any significant temporal autocorrelation that may be existed in a dataset, one of the first remedial measures should be to investigate the omission of one or more of the explanatory variables, especially variables that are related to time. Assuming that, the three time variables in the datasets (month, weekday, hour) have potential influence on the dependent variable, then they are unlikely to be removed from the analysis. Hence, the next step is to apply a differencing procedure to all time independent variables in the dataset to convert them into their differences values. The differencing procedure can be applied by subtracting the previous observation from the current observation, as shown in Equation (13) [20]:

$$
D\left(Y_{t}\right)=Y_{t}-Y_{t-1}
$$

where,

$D(Y)$ : the difference of variable $Y$ at lag $t$,

$Y_{i}$ the value of $Y$ at lag $t$,

$Y_{t-1}$ : the value of $Y$ at lag $t-1$.

The rho (i.e. the residual autocorrelation coefficient) is assumed to be (1.0) in the differencing procedure, which could overestimate the true rho value [21]. The first order differencing is applied to the I-70 (2014) dataset, and the ordinary least square residuals were obtained, then the Durbin-Watson $(D W)$ test is calculated to check for the temporal autocorrelation. The result of the $D W$ statistic showed that the temporal autocorrelation was still existed even after applying the first order differencing. Although the first order differencing is enough to show whether the differencing procedure can be used to remove the serial (temporal) correlation or not [21], however, more differencing orders (up to 7 orders) are applied to the I-70 (2014) dataset, and the Durbin-Watson test ( $D W$ statistic) is calculated each time to check for the temporal autocorrelation. The results showed that the temporal autocorrelation was not removed by this method. Table 3 shows seven differencing orders that were applied to the data and their $D W$ statistics.

Table 3. Differencing results for 2014 I-70 data.

\begin{tabular}{ccccc}
\hline Difference order & DW statistic & $\begin{array}{c}\text { Auto correlation } \\
\text { coefficient }\end{array}$ & p-value & Decision \\
\hline D1 & 1.841 & 0.0731 & 0.0002 & sig. \\
D2 & 1.833 & 0.0724 & 0.0001 & sig. \\
D3 & 1.831 & 0.0722 & 0.0001 & sig. \\
D4 & 1.823 & 0.0812 & 0.0001 & sig. \\
D5 & 1.821 & 0.0822 & 0.0001 & sig. \\
D6 & 1.829 & 0.0781 & 0.0001 & sig. \\
D7 & 1.820 & 0.0825 & 0.0001 & sig. \\
\hline
\end{tabular}




\subsection{The Cochrane-Orcutt Procedure}

When the differencing procedure cannot eliminate the temporal autocorrelation in a dataset, then the Cochrane-Orcutt procedure should be applied for the Autoregressive AR (1) term of this dataset [20]. The procedure uses the ordinary least square residuals to obtain the value of rho which minimizes the sum of squared residuals. Rho is then used to transform the observations of the variables. The process continues until convergence is reached [20] [22]. Considering the general ordinary least squared regression model:

$$
Y_{t}=\alpha+X_{t} \beta+\varepsilon_{t}
$$

where,

$Y_{t}$ the dependent variable at time (lag) $t$,

$\alpha$ : the intercept,

$\beta$ : the vector of regression coefficients,

$X_{t}$ the vector of explanatory variables at time (lag) $t$,

$\mathcal{E}_{t}$ : the error term of the model at time (lag) $t$.

When applying the DW test, if the $(D W)$ statistic revealed that the temporal autocorrelation exists among the model error terms, then the residuals must be modeled for the first order autoregressive term AR (1) such that:

$$
\varepsilon_{t}=\rho \varepsilon_{t-1}+e_{t}
$$

where,

$\rho$ : the temporal autocorrelation coefficient (rho) between pairs of observations, $0<\rho<1$,

$e_{t}$ : the error term of the residuals at time (lag) $t$.

The Cochrane-Orcutt procedure is obtained by taking a quasi-differencing or generalized differencing, such that the sum of squared residuals is minimized [20] [22]:

$$
Y_{t}-\rho Y_{t-1}=\alpha(1-\rho)+\beta\left(X_{t}-\rho X_{t-1}\right)+e_{t}
$$

The Cochrane-Orcutt iterative procedure starts by obtaining parameter estimates by the ordinary least square regression (OLS). Applying Equation (15), the OLS residuals are then used to obtain an estimate of rho from the OLS regression. This estimate of rho is then used to produce transformed observations, and parameter estimates are obtained again by applying OLS to the transformed model. A new estimate of rho is computed and another round of parameter estimates is obtained. The iterations stop when successive parameter estimates differ by less than 0.001 [20].

The iterative Cochrane-Orcutt procedure was applied to the I-70 (2014) dataset, and an optimized rho (i.e. the residual autocorrelation coefficient) value of 0.07333 was obtained using the Stata 14 software that minimizes the estimated sum of squared residuals (ESS), then the $D W$ statistic was calculated for the transformed residuals. The results showed that the temporal autocorrelation was removed from the I-70 (2014) dataset, as shown in Table 4. The $D W$ statistic for the I-70 (2014) dataset is changed after applying the Cochrane-Orcutt procedure from 1.843 (with a significant $p$-value of 0.0002 ) to 1.992 (with a non-significant 
$p$-value of 0.7167).

After removing the temporal autocorrelation from the I-70 (2014) dataset, the $D W$ test and the $L M$ test were applied for the aggregated three years' period (2013-2015) for the I-70 dataset. The $D W$ statistic for the three years' period (2013-2015) is 1.971 with temporal autocorrelation of $1.47 \%$ for the I-70 dataset, which is non-significant, as shown in Table 5.

The $L M$ value for the aggregated three years' period (2013-2015) using 36 lags is 41.203 for the I-70 dataset, which is non-significant, as shown in Table 6. The results from the $D W$ test and the $L M$ test indicate that there is no significant temporal autocorrelation among each of the temporal independent variables (i.e. month, weekday, and hour) in the (2013-2015) dataset.

\subsection{The LBQ Test Results}

The Box-Ljung $Q$ statistic $(L B Q)$ is applied to the aggregated three-year period (2013-2015). Table 7 shows the Box-Ljung $Q$ statistic, the auto correlation function $(A C F)$ and the partial autocorrelation function $(P A C F)$ with their $p$-values for the I-70 dataset for the first 36 lags. It can be seen that the LBQ statistic, the $A C F$, and the PACF for all 36 lags are non-significant for the I-70 crash data. The $L B Q$ statistic increases with the lag progress, indicating no temporal autocorrelation within the dataset and confirming the results of the $D W$ test and the $L M$ test.

\section{Conclusion}

Temporal autocorrelation (also called serial correlation) refers to the relationship between successive values (i.e. lags) of the same variable. Although it is a major concern in time series models, however, it is very important to be checked in crash data modeling as well. The results of crash data modeling can be improved

Table 4. Cochrane-Orcutt results for 2014 I-70 crash data.

\begin{tabular}{cccccc}
\hline Iteration \# & rho & ESS & DW & p-value & Decision \\
\hline 1 & 0.07295 & 568.242 & & & \\
2 & 0.07333 & 568.241 & 1.992 & 0.7167 & non-sig \\
3 & 0.07333 & 568.241 & & & \\
\hline
\end{tabular}

Table 5. Overall DW statistic for I-70 crash data.

\begin{tabular}{ccccc}
\hline \multirow{2}{*}{ Year } & Durbin-Watson DW & Autocorrelation Coefficient & p-value & Decision \\
\cline { 2 - 4 } & 1.971 & 0.0147 & 0.1289 & non-sig \\
\hline
\end{tabular}

Table 6. Overall LM statistic for I-70 crash data.

\begin{tabular}{cccc}
\hline & LM statistic & p-value & Decision \\
\cline { 2 - 4 } & 41.203 & 0.2534 & non-sig \\
\hline
\end{tabular}


Table 7. LBQ test results for I-70 crash data.

\begin{tabular}{|c|c|c|c|c|}
\hline Lag \# & $\mathrm{ACF}$ & PACF & LBQ-statistic & p-value \\
\hline 1 & 0.015 & 0.015 & 1.2720 & 0.259 \\
\hline 2 & -0.009 & -0.009 & 1.7093 & 0.425 \\
\hline 3 & -0.024 & -0.024 & 5.1985 & 0.158 \\
\hline 4 & 0.021 & 0.021 & 7.7212 & 0.102 \\
\hline 5 & -0.006 & -0.007 & 7.9130 & 0.161 \\
\hline 6 & -0.013 & -0.013 & 8.9711 & 0.175 \\
\hline 7 & 0.016 & 0.018 & 10.564 & 0.159 \\
\hline 8 & 0.018 & 0.017 & 12.576 & 0.127 \\
\hline 9 & 0.001 & 0.001 & 12.588 & 0.182 \\
\hline 10 & -0.002 & -0.000 & 12.608 & 0.246 \\
\hline 11 & -0.001 & -0.001 & 12.612 & 0.319 \\
\hline 12 & -0.013 & -0.013 & 13.555 & 0.330 \\
\hline 13 & 0.011 & 0.012 & 14.215 & 0.359 \\
\hline 14 & -0.007 & -0.007 & 14.469 & 0.415 \\
\hline 15 & 0.008 & 0.008 & 14.876 & 0.460 \\
\hline 16 & -0.022 & -0.022 & 17.683 & 0.343 \\
\hline 17 & 0.006 & 0.006 & 17.875 & 0.397 \\
\hline 18 & 0.003 & 0.003 & 17.937 & 0.460 \\
\hline 19 & -0.001 & -0.002 & 17.946 & 0.526 \\
\hline 20 & 0.002 & 0.003 & 17.963 & 0.590 \\
\hline 21 & 0.003 & 0.003 & 18.011 & 0.648 \\
\hline 22 & 0.012 & 0.011 & 18.804 & 0.657 \\
\hline 23 & -0.010 & -0.010 & 19.441 & 0.675 \\
\hline 24 & -0.018 & -0.017 & 21.297 & 0.621 \\
\hline 25 & -0.025 & -0.024 & 24.926 & 0.467 \\
\hline 26 & -0.019 & -0.020 & 27.163 & 0.401 \\
\hline 27 & -0.017 & -0.017 & 28.857 & 0.368 \\
\hline 28 & -0.005 & -0.006 & 29.012 & 0.412 \\
\hline 29 & -0.005 & -0.005 & 29.160 & 0.457 \\
\hline 30 & 0.011 & 0.010 & 29.869 & 0.472 \\
\hline 31 & -0.006 & -0.005 & 30.071 & 0.514 \\
\hline 32 & -0.028 & -0.028 & 34.843 & 0.334 \\
\hline 33 & 0.002 & 0.005 & 34.877 & 0.379 \\
\hline 34 & 0.029 & 0.030 & 39.955 & 0.223 \\
\hline 35 & 0.018 & 0.016 & 41.843 & 0.198 \\
\hline 36 & 0.000 & 0.002 & 41.843 & 0.232 \\
\hline
\end{tabular}

when several years of crash data are utilized in the analysis, such as a period of three years instead of one year. However, this means that the same roadway will generate multiple observations over time, which could be correlated due to some temporal (time) component and could adversely affect the precision of parameter estimates. There are several methods that can be used to detect the existence of the temporal autocorrelation in the crash dataset, such as: 1) the residuals 
scatter plots; 2) the Durbin-Watson $(D W)$ test; 3) the Durbin $h$ test; 4) the Breusch-Godfrey ( $L M$ ) test; 5) the Ljung-Box $Q(L B Q)$ test; and 6) correlograms. The residuals scatter plots and correlograms are not formal tests, and they would only suggest whether temporal autocorrelation may exist within crash data. The Durbin $h$ test can only be used when there is a lagged dependent variable in the data. This paper used the Durbin-Watson $(D W)$, Breusch-Godfrey $(L M)$, and the $L B Q$ tests to detect the temporal autocorrelation among the temporal independent variables in the crash data (i.e. hour, weekday, month) for the interstate I-70 in Missouri for the years (2013-2015). Although the applications of these tests can be found in time series models, they have not been addressed in modeling crash data. As such, this paper thoroughly investigated the applicability of these tests to crash data.

\section{References}

[1] King, M.L. (1981) The Alternative Durbin-Watson Test: An Assessment of Durbin and Watson's Choice of Statistic. Journal of Econometrics, 17, 51-66.

[2] Anderson, T.K. (1984) On the Theory of Testing Serial Correlation. Accident Analysis and Prevention, 31, 88-116.

[3] King, M.L. (1983) The Durbin-Watson Test for Serial Correlation: Bounds for Regressions Using Monthly Data. Journal of Econometrics, 21, 357-366.

[4] Lord, D. and Mannering, F. (2010) The Statistical Analysis of Crash Frequency Data: A Review and Assessment of Methodological Alternatives. Accident Analysis and Prevention, 44, 291-305.

[5] Washington, P., Karlaftis, G. and Mannering, F. (2010) Statistical and Econometric Methods for Transportation Data Analysis. 2nd Edition, Chapman Hall CRC, Boca Raton.

[6] Savolainen, P., Mannering, F., Lord, D. and Quddus, M. (2011) The Statistical Analysis of Highway Crash-Injury Severities: A Review and Assessment of Methodological Alternatives. Accident Analysis and Prevention, 43, 1666-1676.

[7] Cameron, A.C. and Trivedi, P.K. (1998) Regression Analysis of Count Data. Cambridge University Press, Cambridge. https://doi.org/10.1017/СBO9780511814365

[8] Caliendo, C., Guida, M. and Parisi, A. (2007) A Crash-Prediction Model for Multilane Roads. Accident Analysis and Prevention, 39, 657-670.

[9] Greene, W. (2012) Econometric Analysis. 7th Edition, Prentice Hall, Upper Saddle River.

[10] Gujarati, D. (1992) Essentials of Econometrics. McGraw-Hill, New York.

[11] Miaou, S.P., Song, J.J. and Mallick, B.K. (2003) Roadway Traffic Crash Mapping: A Space-Time Modeling Approach. Accident Analysis and Prevention, 6, 33-57.

[12] Lord, D. and Bonneson, A. (2007) Development of Accident Modification Factors for Rural Frontage road Segments in Texas. Transportation Research Record, 2023, 20-27. https://doi.org/10.3141/2023-03

[13] Hilbe, J. (2014) Modeling Count Data. Cambridge University Press, Cambridge. https://doi.org/10.1017/cbo9781139236065

[14] Thomas, R.L. (1993) Introductory Econometrics: Theory and Applications. 2nd Edition, Longman, London.

[15] Studenmund, A.H. (2001) Using Econometrics-A Practical Guide. Addison-Wes- 
ley-Longman, London.

[16] Box, G.E.P., Jenkins, G.M. and Reinsel, G.C. (1994) Time Series Analysis-Forecasting and Control. Prentice Hall, Englewood Cliffs.

[17] Chatfield, C. (1996) The Analysis of Time Series-An Introduction. 5th Edition, Chapman and Hall CRC, London.

[18] Warner, R.M. (1998) Spectral Analysis of Time-Series Data. Guilford Press, New York.

[19] Tsay, R.S. (2010) Analysis of Financial Time Series. Wiley \& Sons, Hoboken. https://doi.org/10.1002/9780470644560

[20] Wooldridge, M. (2013) Introductory Econometrics: A Modern Approach. 5th Edition, South-Western, Mason.

[21] Pindyck, R.S. and Rubinfeld, D.L. (1981) Econometric Models and Economic Forecasts. McGraw-Hill, New York.

[22] Cochrane, D. and Orcutt, G.H. (1949) Application of Least Squares Regression to Relationships Containing Autocorrelated Error Terms. Journal of the American Statistical Association, 44, 32-61.

Submit or recommend next manuscript to SCIRP and we will provide best service for you:

Accepting pre-submission inquiries through Email, Facebook, LinkedIn, Twitter, etc. A wide selection of journals (inclusive of 9 subjects, more than 200 journals)

Providing 24-hour high-quality service

User-friendly online submission system

Fair and swift peer-review system

Efficient typesetting and proofreading procedure

Display of the result of downloads and visits, as well as the number of cited articles

Maximum dissemination of your research work

Submit your manuscript at: http://papersubmission.scirp.org/

Or contact jtts@scirp.org 